\title{
A clinical, pathological, and genetic study of an unusual form of Tay-Sachs disease with macular degeneration in the family ${ }^{1}$
}

\author{
PAUL R. DYKEN ${ }^{2}$ AND WOLFGANG ZEMAN \\ From the Departments of Neurology (Pediatric Neurology) and Pathology, Indiana \\ University of School Medicine, Indianapolis, Indiana, U.S.A.
}

This paper describes a non-Jewish patient, with the classical features of Tay-Sachs disease, who exhibited several unusual clinical and genetic deviations.

\section{CASE SUMMARY}

HISTORY A 17-month-old white male was admitted on 7 March 1963 because of poor development, deterioration in motor and mental abilities, and intestinal disturbances. The informants were the parents who were intelligent and reliable. Further information was obtained from the family physician. The infant also had feeding difficulties, poor muscle tone and head control, and displayed wandering eye movements.

The patient was the product of a full-term pregnancy, complicated in the first trimester by a threatened abortion. The delivery was uneventful. No significant abnormalities were observed neonatally, although a 'mongol spot' over the back and a capillary haemangioma on the right hand were recorded. The infant fed vigorously, but for the first three and a half months of life was irritable, especially at night, presumably because of intermittent flatus and abdominal distention. Diarrhoea was an occasional problem for the next five months and was thought to be a secondary reaction to the feeding formula. At 3 months the infant had varicella, at 5 months urticaria, and at 6 months roseola. Moreover, he had frequent minor 'colds', characterized primarily by alternating nasal obstruction and drainage.

At 6 months the infant was bright-eyed and noticed his parents and surroundings. He had fair head control, rolled over from the back and from the abdomen, was able to sit if propped up by pillows, and with help he could stand. At this time he fell from his crib. Although he was not injured, he developed no new skills thereafter. Between this event and admission he lost completely the abilities to roll over and to sit up for more than seconds at a time. When attempts were made to make him stand, he would stiffen the legs. The parents noticed searching eye movements for two months before admission. He no

${ }^{1}$ This study was supported by a research grant from the United Health Foundation, Inc., Elkhart, Indiana, U.S.A.

${ }^{2}$ Present address: Department of Neurology, 660 South Kingshighway, St. Louis, Missouri. longer seemed interested in his surroundings and did not respond to objects that previously attracted his attention. He became extremely irritable, and the slightest noise would make him jump and shake for a few seconds. Feeding became increasingly difficult; mashed foods were not tolerated well, and bottle feeding was excessively prolonged. Red blotches were observed on his face during and immediately after eating. He frequently gagged when feeding. He had no convulsions, jaundice, peripheral oedema, or vomiting.

PHYSICAL FINDINGS At the time of admission he was $80 \mathrm{~cm}$. long and weighed $8 \cdot 3 \mathrm{~kg}$. The vital signs were normal. General inspection revealed a normal-sized infant with puffiness around the eyes, flattened nose, and protuberant lips. The skin had a peculiar velvety texture and was diffusely bronzed as if the infant had been out in the sun. A bluish-brown-gray 'mongol spot' measuring $3 \times 3 \mathrm{~cm}$. was seen just to the left of the midline in the lumbosacral area. Many small, slightly elevated, pigmented naevi were present, particularly over the head. A bright red, easily blanched capillary haemangioma with irregular edges, measuring $2 \times 1 \mathrm{~cm}$., was present on the right hypothenar eminence. No scars, bruises, or other skin marks were seen anywhere on the body. Hair distribution and texture was normal.

The occipito-frontal circumference was $48 \mathrm{~cm}$. and the anterior fontanelle was slightly open and tight but not bulging. A highly resonant tympanitic sound could be elicited with percussion over the cranium. The cephalic veins were not engorged. A good deal of nasal discharge was present and from time to time the baby breathed sonorously. The abdomen was protuberant but on careful palpation, while the infant was relaxed, no abnormalities were detected. The liver was barely palpable below the right costal margin and was normal. The spleen and kidneys were not palpable. No ascites was present. The colon was filled with a great deal of flatus and the ampulla recti contained small, hard faeces. The infant had been circumcised. There was a small reducible, indirect, inguinal hernia on the left. The heart and lungs were normal and no other abnormalities were detected on careful general physical examination.

The baby appeared lethargic, yet noise or tactile stimulation caused an exaggerated startle response and 


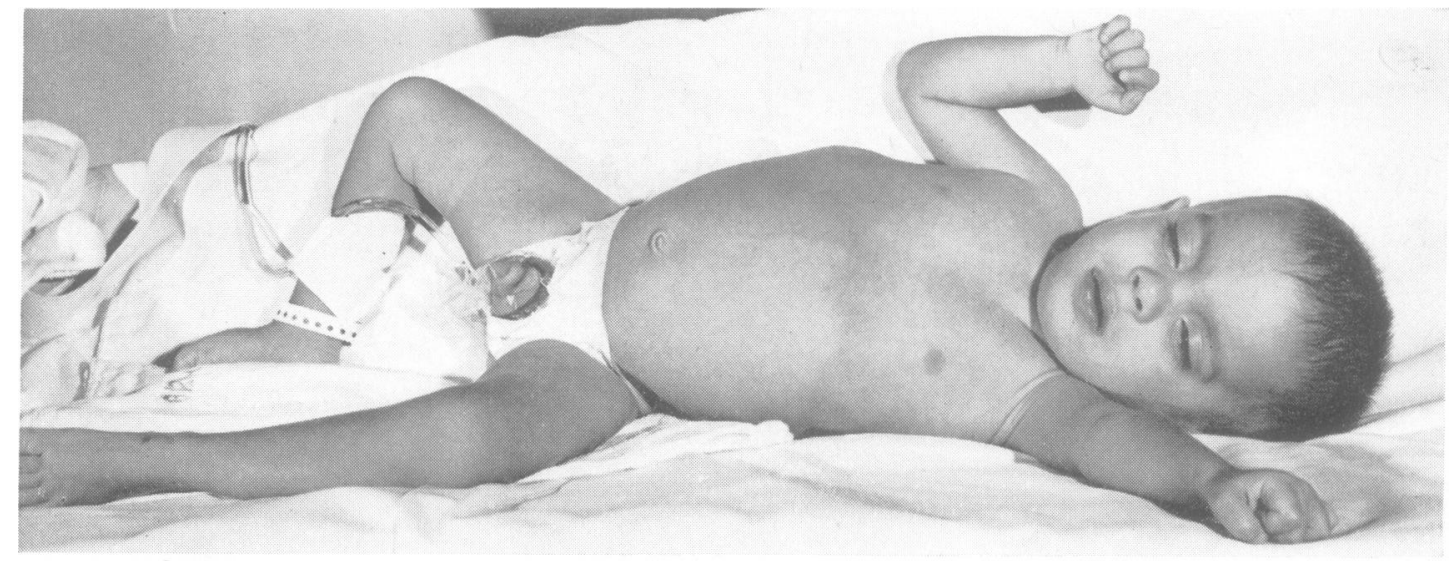

FIG. 1. Patient demonstrating fencing reaction after auditory stimulus. The fencing reaction consists of the following sequence: extension of the left elbow, abduction of the left shoulder, flexion of the right elbow, abduction of the right shoulder, extension and then flexion of both lower extremities with head turning to the left. This reaction was associated with a high-pitched cry. Note facial characteristics and abdominal distention.

high-pitched crying. Unless bothered he lay quietly, but on occasion would cry and posture spontaneously. Although all extremities were spastic and paretic, voluntary movements were observed. Bilateral extensor toe signs were elicited, and with palmar stimulation there was a pronounced mental reflex. Superficial abdominal reflexes were hypoactive. Head control was poor and the infant was unable to sit or roll over. He responded to light touch, pinprick, and vibratory stimuli at all areas, often in the form of a fencing reaction (see Fig. 1). On occasion, this posture was assumed spontaneously, but was regularly observed in response to sudden noises. The eyes exhibited wandering, intermittently dyscon-

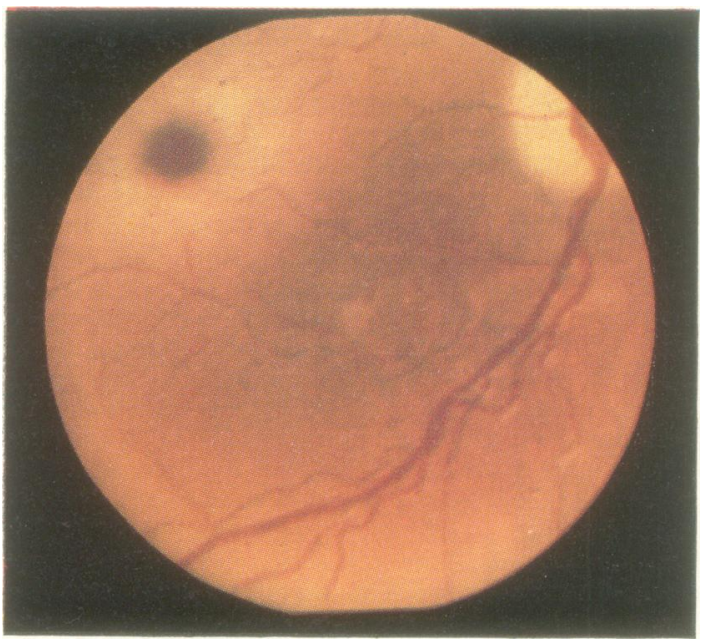

FIG. 2. Macular changes of Tay-Sachs disease in left fundus of proband. Similar changes are present in right eye. jugate movements. He would gag when attempting to swallow. The optic fundi showed changes compatible with Tay-Sachs disease, depicted in Fig. 2 as a sharply demarcated 'raspberry' red spot surrounded by a halo웅 of greyish-white oedematous retina. Although both option discs were pale and atrophic, pupillary light reflexes were present. Choroidal vessels were easily visualized. The $T$ retinal arteries and veins were normal. The peripheral part of the retina appeared somewhat oedematous.

COURSE IN HOSPITAL During his stay in hospitake excessive, intermittent nasal secretions made oral feeding difficult. Placed on a pureed diet, supplemented by mashed and blended foods with a high protein content, the baby's weight increased to $9.5 \mathrm{~kg}$. in 27 days.

The results of studies not mentioned in the text can be found in Table I. The infant had a persistent eosinophilia varying from $14 \%$ (routine differential) to $85 \%$ (Randolph total eosinophil count) in the face of an otherwise essentially normal leucocyte count (Table II). The fluctuation in the eosinophilia was not dependent on the clinical condition of the child and bore no relation to nasal or respiratory symptoms. The child had been placed on sulphasoxazole (Gantrisin) for a short period, but the eosinophilia was present before and remained long after it was discontinued. An attempt was made to discover the cause of the eosinophilia. The proband had negative skin tests for tuberculosis, histoplasmosis, blastomycosis, coccidiomycosis, and trichinella. Several stools were examined for ova and parasites, and all were negative. A toxoplasmosis dye test was negative. Blood cortisol, urinary 17 -ketocorticosteroids, and urinary 17-hydroxycorticosteroids were determined and found to be within normal limits for this age group.

Vacuolated lymphocytes comprised $12 \%$ and $8 \%$ of the total lymphocyte and monocyte count. Special stains on several blood smears were performed, using techniques employed by Spiegel-Adolf, Baird, Coleman, and 
TABLE I

LABORATORY ANALYSIS

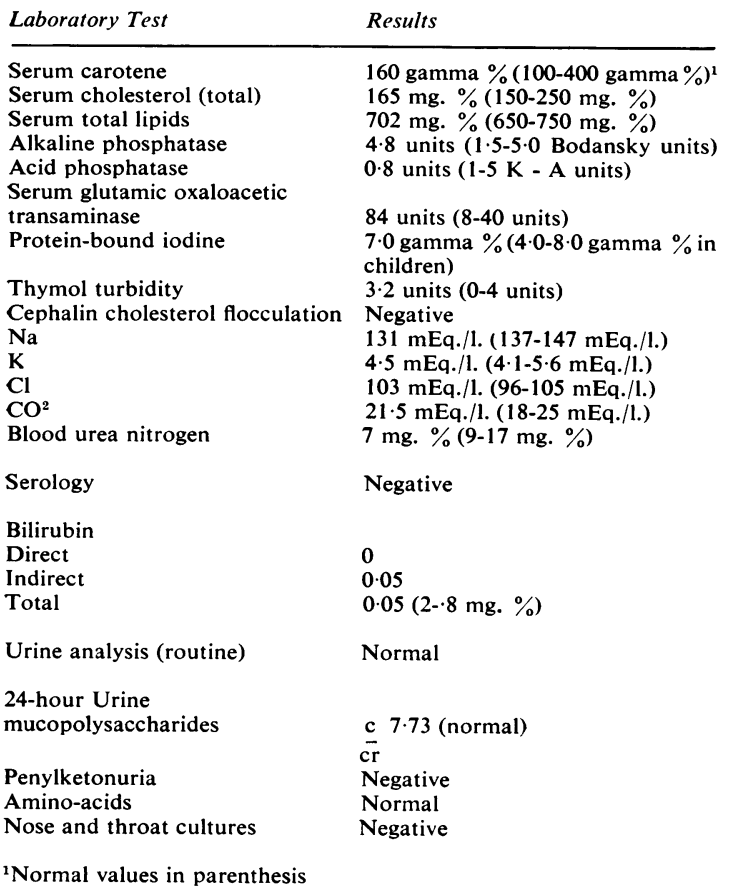

Szekely (1962) but no cytoplasmic or nuclear inclusions similar to those reported by them were seen. Smears stained for acid phosphatase, adenosine triphosphatase, and esterase were unremarkable when compared with normal controls. The eosinophils stained in no unusual manner with Giemsa, Wright, Sudan Black B, Oil Red O, or P.A.S., and for acid phosphatase, adenosine triphosphatase, or esterase activity.
Analysis of a bone marrow aspiration was as follows: 'On microscopic examination there was good cellularity and, as was noted in the peripheral blood, there was a rather striking increase in normal-appearing eosinophils. Monocytes were somewhat increased but were qualitatively normal. A careful search was made for possible inclusion-bearing histiocytes and no cells showing such a histiological picture were detected. There were no leukaemic changes or tumour cells.' Slides were obtained at the bone marrow aspiration for further enzyme and fat stains. No significant abnormalities were seen in Sudan Black B, Oil Red O, P.A.S., or acid phosphatase preparations.

Radiographs of the chest, vertebrae, and hands showed some generalized demineralization of the bony structures but no specific abnormalities. The skull radiograph exhibited spreading of the sutures. The head measured within the upper limits of normal in length and breadth but there was a definite increase in the height of the skull. The sella turcica was not remarkable. There was questionable depression of the olfactory plate. A pneumoencephalogram showed a normal, if not small, ventricular system. The lateral ventricles, third ventricle, fourth ventricle, and cerebral sulci were well demonstrated. A patent cisterna veli interpositi was visible. The basilar cisterns were larger than normal.

Serial electroencephalograms (E.E.G.) were compatible with degeneration of the central nervous system. The first E.E.G. showed 3 to 5 cycle per second dysrhythmia of a synchronous nature. Superimposed synchronous spike activity was a later feature.

Protein electrophoresis done on several occasions was normal except for mild elevations in the alpha 1 and 2 fraction in the serum and a slight elevation of the alpha globulin fraction in the cerebrospinal fluid (compared with the normal values of Dunn and Pearce, 1961).

A brain biopsy was performed after the indications had been carefully weighed. Upon opening the dura in a subtemporal craniotomy, pale yellow, glistening, firm brain tissue bulged through the hole. A $1 \mathrm{~cm}^{3}$ sample was

TABLE II

HAEMATOLOGY

Haematocrit
Haemoglobin
White cell counts
Bands
Adult neutrophils
S. lymphocytes
Monocytes
Eosinophils
Basophils

$\begin{array}{rrr}41 & 33 & 32 \\ 13 \cdot 3 & 9 \cdot 6 & 9 \cdot 4 \\ 18,600 & 10,700 & 13,050 \\ 0 & 0 & 0 \\ 21 & 13 & 21 \\ 64 & 43 & 50 \\ 0 & 5 & 9 \\ 14 & 39 & 25 \\ 1 & 0 & 0\end{array}$

Randolph absolute eosinophil counts (percentage of simultaneous total leucocyte counts in parentheses)

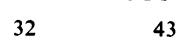

$$
9 \cdot 4
$$

3,050
0
21
50
9
25
0

一

$6,040(60 \%) 11,300(86 \%)$

$-$

$7,400(53 \%)$

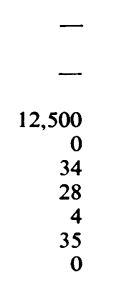

42
$12 \cdot 3$
14,100
0
61
15
13
11
0

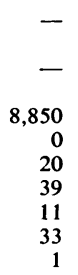

Vacuolated lymphocytes (\% of total lymphocyte count, with 200 cells counted)

Blood typing and $\mathrm{Rh}$ factor 


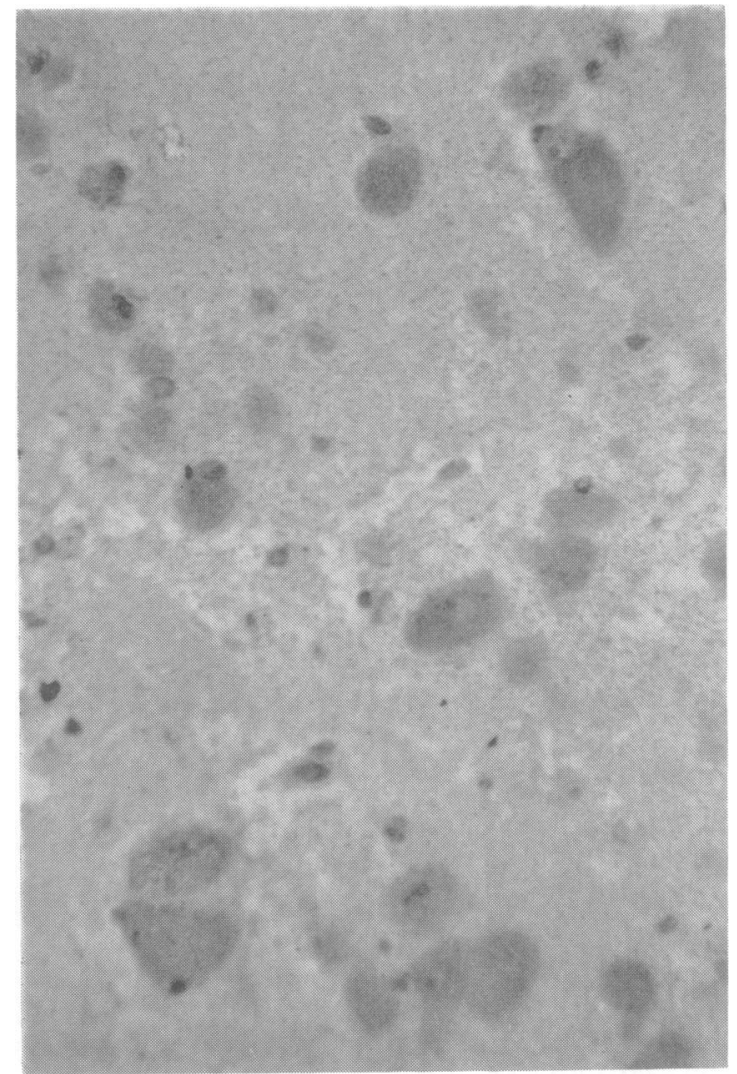

FIG. 3. Cerebral cortex from right superior temporal gyrus showing distended neuronal perikarya. Acidified cresyl violet. $\times 225$. The material is P.A.S. positive.

excised and immediately prepared for electron-microscopic, microchemical, histochemical, and routine histological studies. At this time it was noted that the cortex, although extremely pale, was approximately 5 to $8 \mathrm{~mm}$. in thickness. Because of the bulging brain the fascia and dura mater were left open and the skin closed. A lowered haemoglobin value before operation required a total of $500 \mathrm{ml}$. of whole blood during and after the procedure.

The results of the biopsy study will be reported elsewhere in detail. In brief, all neuronal perikarya displayed the typical ballooning of Tay-Sachs disease (Fig. 3). The intraganglionic lipid was not sudanophilic in paraffinembedded sections (Fig. 4) but slightly so in unfixed material. Under phase-contrast microscopy, numerous organelles measuring approximately 1 to $2 \mu$ in diameter and spherical in shape were seen. In histochemical preparations, demonstrating a variety of dehydrogenases, reactive astrocytes in the cortex and subcortical white matter were conspicuously stained, whereas the perikarya often appeared like cut-out holes. Only glucose-6phosphate dehydrogenase gave a diffuse staining of the neuronal soma in which the unstained lipid granules appeared like translucent fish eggs, densely packed. Techniques for the demonstration of alkaline phosphatase gave normal results, but acid phosphatase, as demonstrated by the Gomori technique, was highly active. With the Barka technique, using pararosanolin as a coupling dye, activity in neuronal perikarya was rather weak in comparison with vascular endothelial cells and perivascular macrophages.

Electron miscroscopic observations displayed huge quantities of cytoplasmic organelles (Fig. 5) reminiscent of, but not entirely similar to, the membranous cytoplasmic bodies described by Terry and Korey (1960) and by Terry, Korey, and Weiss (1962). Further studies are still in progress.

\section{OBSERVATIONS IN THE FAMILY}

A family survey was made (see Fig. 6). No Jewish ancestry or consanguinity was detected.

THE MOTHER The mother had had three previous pregnancies and one following the birth of the patient. Two of them ended in abortion before the third month. She had always been in excellent health and had maintained frequent pre-natal checks which were unremarkable. She had acne as an adolescent and during this time developed a bronze mottling of the face which persisted. Otherwise the physical examination was com $\vec{\Phi}$ pletely within normal limits. An E.E.G. was interpreteo as normal.

SISTER The patient's 7-year-old sister made good marks in school and seemed to be an unusually intelligent girlo She was in excellent health except for the usual childhook diseases and occasional 'colds' which necessitated in jections from the family physician. She also had symptomst of allergies. The physical examination was normal. The E.E.G. was interpreted as normal.

BROTHER The patient's 4-month-old brother was the product of an uneventful but hormone-treated full-term pregnancy and normal delivery. He also suffered from intermittent abdominal distention, excess flatus, occasional alternating diarrhoea and constipation, and some 'spitting up'. In addition, he had had several 'colds'. Nevertheless, he was a very vigorous and active child who showed good head control and was deemed quite strong by everyone who had any contact with him. Before the time of the examination, the infant had been placed on broad-spectrum antibiotics because of a respiratory infection. He was irritable and had diarrhoea. At the time of the physical examination he smiled appropriately and recognized his mother quickly. The muscle tone was good. A 'mongol spot' measuring $2 \times 2 \mathrm{~cm}$. was observed just to the left of the midline in the lumbar area. He had good head control and in fact seemed strong compared with others in this age group. A startle response was present but was not associated with any increase in reactivity or abnormal postures. The occipito-frontal circumference was $43 \mathrm{~cm}$. and the head was symmetrical. A tympanitic sound was heard with percussion over the head. The anterior fontanelle was tight but not bulging. The plantar 


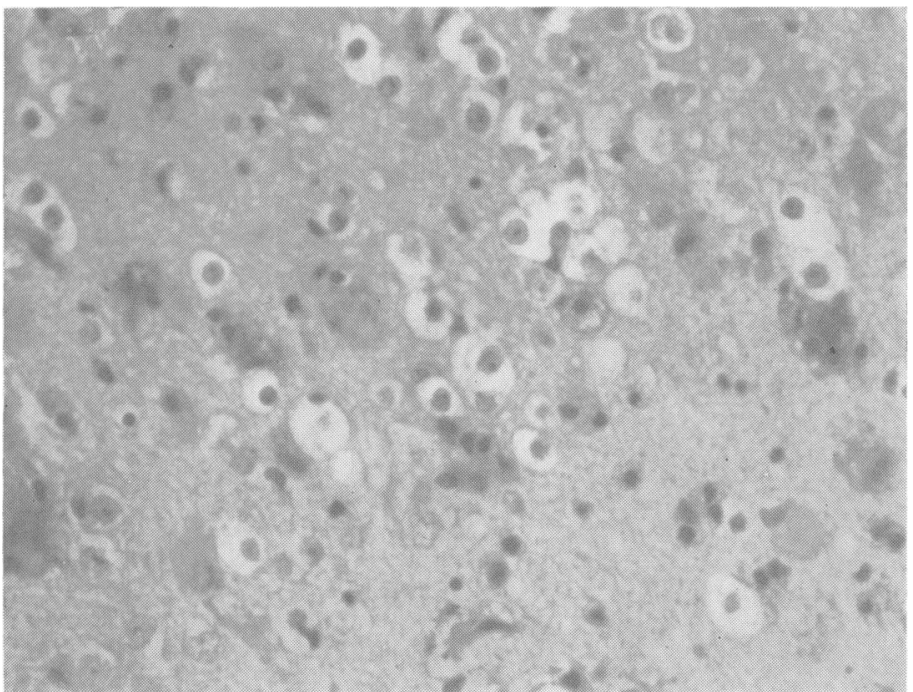

FIG. 4. Cortical biopsy specimen showing ballooned nerve cells with the cytoplasm appearing 'empty' because of the elution of lipid granules during embedding. Vascular endothelial cells and astrocytes, on the other hand, have retained the P.A.S.-positive lipid granules. $\times 240$.

FIG. 5. Cortical perikaryon. A segment of the nucleus is shown on the upper left, the Golgi apparatus is distended, and the cytoplasm contains numerous membranous cytoplasmic bodies characteristic of Tay-Sachs disease. Fixation in buffered osmium tetroxide, embedded in methacrylate and lead stained. Approximately $\times 28,000$.

FIG. 4

FIG. 5

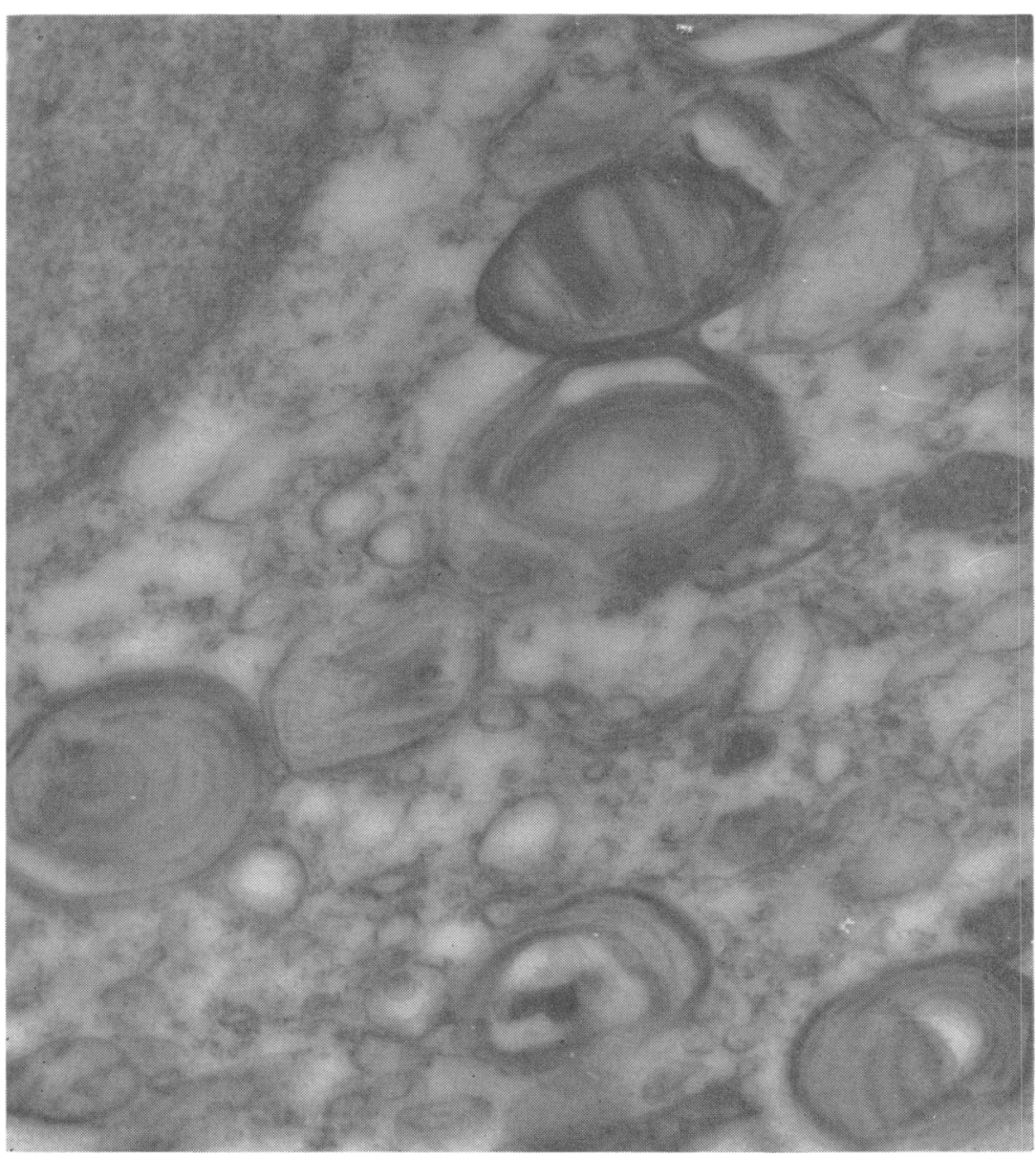




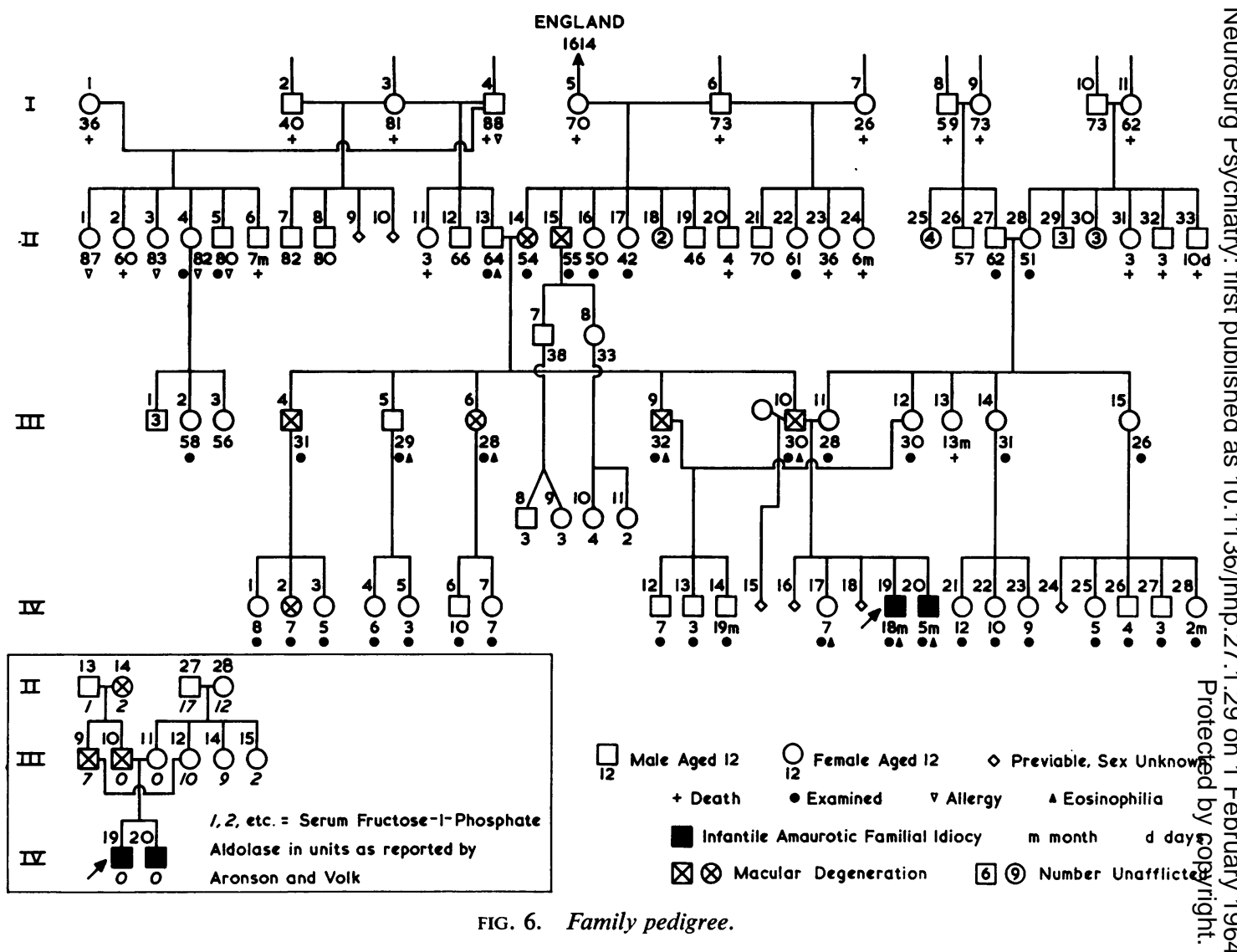

response was extensor bilaterally. The myotatic reflexes could only be elicited at the patella and biceps and were physiological. Sucking was good and no abnormal responses could be elicited. The eye movements and pupillary reactions were normal. The optic fundi showed bilateral macular changes compatible with Tay-Sachs disease and identical with the findings in the proband except for a lighter cherry-red central area. No optic atrophy or other peculiar features were visualized in detailed examinations. An E.E.G. at 4 months of age was within normal limits but a second E.E.G. at 5 months of age was abnormal, showing diffuse 3 to 5 cycle per second dysrhythmic slowing which was slightly worse on the right.

THE FATHER The father was 30 years old. The present marriage was his second, the first ending in divorce after the wife aborted at 3 months' gestation. He gave a history of having had the usual childhood diseases and at 2 years of age severe pneumonia. He also was afflicted with various allergies in the form of rhinitis, sinusitis, asthma and urticaria, beginning severely at the age of 23 years. He was found to be allergic to dust, tobacco, paints, wool, animal hair, beer hops and yeast, among other things. Although previous eye examinations were said to be normal, in 1956 while applying for a job which required a high amount of visual acuity he was told he had loss of central vision and degeneration of the retina. His $\frac{0}{\circ}$ impaired vision, which was better at night, has incapaci- $\varrho$ tated him to some extent, even though presently he $\overrightarrow{\overrightarrow{0}}$ works regularly in the production line of a battery 3 factory. He has had no significant change in mental or neurological functions, but with the illness of his child, he has become more 'nervous'.

The general physical examination revealed the mucous membranes of the nose to be slightly oedematous and reddened, producing a great deal of secretion. No bony or skin abnormalities were found. He was apprehensive and had mild, intermittent, irregular tremors when his $\delta$ hands and arms were extended for long periods. The $§$ patellar reflexes were exaggerated. Other reflexes were physiological. No agnosias, apraxias, or aphasia were uncovered. Drawing was somewhat difficult to perform, $\frac{D}{2}$ presumably because of the deficits in vision. These suggestions of minimal neurological dysfunction were thought $N$ to be within the range of normal. The optic fundi showed pronounced symmetrical pigmentary macular degenera- $N$ tion (Fig. 7). The E.E.G. was interpreted as normal. 


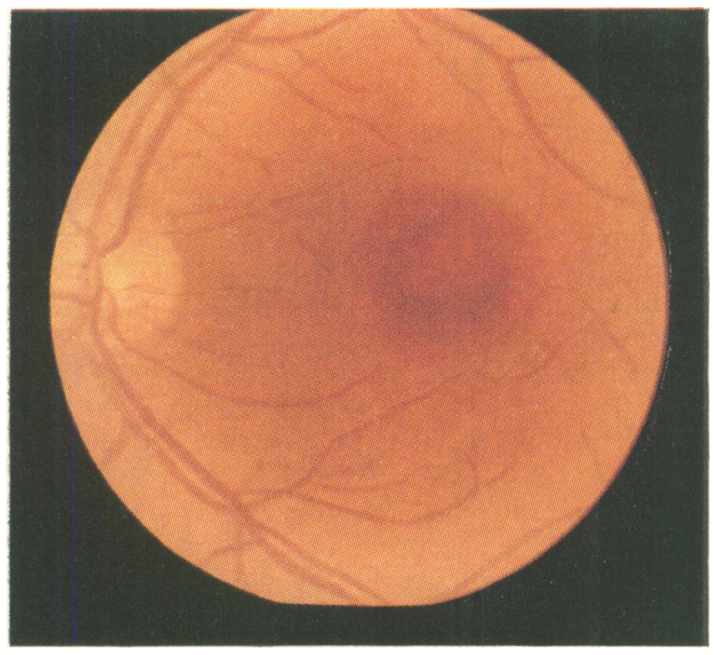

FIG. 7. Degeneration of the macula in left eye of proband's father. Similar changes are present in right eye. Macular degeneration of a similar nature but of different extent found in other members of this family (see Figure 4).

OTHER MEMBERS OF THE FAMILY Thirty-six other members of the family were examined, with particular reference to ophthalmological or neurological abnormalities. The paternal grandmother (II, 14), one paternal great uncle (II, 15), two paternal uncles (III, 4, III, 9), one paternal aunt (III, 6), and one female cousin on the paternal side $(I V, 2)$ had macular degeneration. A paternal uncle (III, 9) with bilateral macular depigmentation of a mild degree, who did not complain of poor vision, gave a history of sensitivity to flickering lights, which he avoided for fear of losing consciousness. At these times he had a momentary strange feeling. He was found to be of average intelligence. Besides the eye findings, the only suggestive neurological finding was exaggerated patellar reflexes. The paternal grandmother (II, 14) had signs and symptoms of hypocalcaemia, resulting from a thyroidectomy 25 years before. She was alert, and she had no subjective visual disturbance, even though there were signs of abnormal macular pigmentation and drusen. No significant related neurological abnormalities were detected in those found to have macular degeneration. The retinal abnormalities involved the macular area, and in all but one individual (III, 4) were bilaterally symmetrical. In some members (II, 14, III, 9, and IV, 2) vision was not appreciably affected at the time of our examination. The age of onset was unknown in those without appreciable visual loss, but in III, 4, III, 6, and the father (III, 10) began in the third decade. It was of interest that many members of the family had some evidence of allergy. No other significant, possibly related diseases or defects were encountered in the members examined. With the exception of the maternal great grandfather (I, 10) who had spells of psychotic behaviour, the unexamined members of the family were free of related neurological or ophthalmological abnormalities. With the help of an interested relative, a pedigree dating back to 1614 and originating in England in one line was obtained.

Differential leucocyte counts were performed on selected members of the family. The mother had no eosinophils but the father $(10 \%$ and $7 \%)$, the younger brother $(4 \%, 4 \%$, and $5 \%)$, two paternal uncles $(3 \%$ in III, 5 and $6 \%$ in III, 9), the paternal aunt (3\%), and the paternal grandfather $(7 \%)$, all had mild to moderate eosinophilia. In the blood of the brother $14 \%$ vacuolated lymphocytes were found, $8 \%$ in the mother, $6 \%$ in the father, and $7 \%$ in the sister.

Serum fructose-1-phosphate aldolase determinations were performed in Dr. Bruno Volk's laboratory on the patient, both parents, and the younger sibling. All were devoid of this circulating enzyme. The analysis of sera from other selected members of the family can be seen in Figure 4.

\section{DISCUSSION}

Certain unusual features about the proband and his family deserve comment. Bilateral macular changes as seen in Fig. 2 have also been reported in NiemannPick and Gaucher's disease (Walsh, 1957). There is little to suggest that the patient suffers from either of these disorders and from the macular changes alone one can assume Tay-Sachs disease to be present. Similar changes in the younger brother, even without overt neurological deficit, suggest that he, too, has infantile amaurotic familial idiocy. This argument is supported by minimal E.E.G. abnormalities and megalencephaly, although Aronson, Lewitan, Rabiner, Epstein, and Volk (1958) consider the latter to be an unusual early manifestation.

Congenital stigmata were found in both the proband and his sibling. The former had a mongol spot, a haemangioma, and a patent cisterna veli interpositi. The latter had similar skin lesions. The significance of these congenital stigmata is unknown.

Another unusual clinical feature was the persistent 'fencing reaction' to tactile or auditory stimulation. This feature disappeared during the course of followup examinations. The fencing reaction seems quite similar to the tonic neck reflex and probably represents a non-specific, possibly unilateral, supranuclear vestibular disturbance secondary to a temporary inequality of the disease process.

The presence of eosinophilia, to our knowledge, has not been previously found to be associated with Tay-Sachs disease. Maruyama, Takeuchi, Tajiri, Harjano, and Makita (1960) reported a patient with infantile amaurotic familial idiocy who had inconsistent eosinophilia of a moderate degree. The familial incidence of allergies in the present pedigree, besides the mild to moderate eosinophilia in those members surveyed, suggests that the eosinophilia in the proband can only partially be explained on this basis. Also, according to Wintrobe, eosinophilia of 
the degree present in the proband is too high to be explained purely on an allergic or familial basis. Furthermore, due to the absence of common causes of pronounced eosinophilia (Wintrobe, 1961) and the normal adrenal cortical function, one may speculate that the eosinophilia in this case represents a unique response of the individual to the abnormal lipids in the various cells of his body. The absence of pronounced eosinophilia in the younger sibling may reflect the lack of significant generalized cerebral disease, or merely a lack of the ability to respond in this way.

The electroencephalographic abnormalities, showing gradual deterioration, are not pathognomonic. When the younger sibling showed bilateral macular changes an E.E.G. was normal. A tracing performed one month afterward, however, was abnormal and suggested early cerebral disease. Electroencephalograms on all other surveyed members of the family were normal.

One of the most unusual features of this family is the seemingly pure macular degeneration of varying onset, morphology, and clinical manifestations in two preceding and one current generation on the paternal side. By current examination and laboratory study there was no significant cerebral disease found in the 39 other members surveyed. The unique occurrence of these two conditions, together in the same family, is remarkable. Reports of other unusual forms of amaurotic familial idiocy are rare. Wyburn-Mason's (1943) cases 8, 9, and 10 are unusual in that one child had infantile amaurotic familial idiocy, a younger sibling had bilateral macular changes of Tay-Sachs disease at 10 years of age, and the mother was afflicted with a deteriorating neurological disease characterized by bilateral optic atrophy and dementia while an adult. Maere and Myle's family included in Van Bogaert's (1962) publication entitled 'Chronic amaurotic idiocy without amaurosis' consisted of a mother, son, and daughter who were afflicted with diseases characterized by 'severe mental disturbances, ataxia, and short stature', who apparently had some form of cerebromacular degeneration, one of which was of an 'infantile type'. The only family, however, which we were able to find that closely resembled ours was that of Monnet, Tommasi, Tissot, and Gaillot (1959) in which the mother and a maternal uncle of a child afflicted with Tay-Sachs disease had 'Stargardt's macular degeneration'.

Whether macular degeneration and Tay-Sachs disease are intimately related to one another or occur purely on a chance basis cannot be definitely stated in the light of current diagnostic studies. Even though serum fructose-1-phosphate aldolase (Aronson, Perle, Saifer, and Volk, 1962) was absent in the father, who has macular degeneration, and in the proband and his sibling who have infantile amaurotic familial idiocy, it was also absent in the mother who has no physical stigmata. These findings seem to be in agreement with current opinion that Tay-Sachs disease is an autosomal recessive determined disease (Kozinn, Wiener, and Cohen, 1957; Myrianthopoulos, 1962; Aronson, Aronson, and Volk, 1959; Aronson and Volk, 1962; Knudson and Kaplan 1962). The activity levels of this enzyme in other members of the family exhibited no correlation with the occurrence of macular degeneration.

The morphological and chemical observations made on the biopsy specimen were generally in agreement with those reported in the literature (Lazarus, Wallace, and Volk, 1962), and with the recent extensive studies of Gomez, Gonatas, Korey, Samuels, Stein, Suzuki, Terry, and Weiss (1963).

The occurrence of Tay-Sachs disease with congenital stigmata in the same patients is interesting and may be significant. No explanation of the association is offered. Further, the occurrence of familial macular degeneration in the paternal ascendants of our two patients with Tay-Sachso disease can only be recorded at this time. The $\tilde{C}$ previous similar observations by Monnet et a $\overrightarrow{\mathbb{N}}$ 음 (1959) suggest more than a coincidental relation.

\section{SUMMARY}

A 17-month-old white male had the onset of abdo minal distention, deterioration in mental and motợ $\overrightarrow{\bar{Q}} \overrightarrow{0}$ capabilities, respiratory difficulties, bronzing of the skin, wandering eye movements, and extreme irritability before the age of 6 months. There was poor mental and motor development, spasticity and hyperreflexia of all extremities, fencing posturing with the slightest sound or tactile stimuli, pronounced signs of corticospinal involvement, megalencephaly, cutaneous abnormalities, and bilateral symmetrical macular changes compatible with Tay-Sachs disease. The infant showed a pronounced, unexplained eosinophilia, deterioration on persistently abnormal E.E.G.s, minimal abnormalities in protein electrophoresis of serum and cerebrospinal fluid, and had no circulating serum fructose-1-phosphate aldolase. Histological, histochemical, electron microscopic, and other laboratory studies were compatible with the clinical diagnosis of Tay-Sachs disease.

A younger sibling was found to have identical macular changes as the proband, a similar abnormal E.E.G., a mild eosinophilia, and megalencephaly.

The retinal abnormalities in other members of the paternal side of the family consisted of mild to severe macular degeneration of varying types. No remarkable neurological abnormalities were de- 
tected. In addition, electroencephalographic studies on selected members of the family were unremarkable. Eosinophil counts showed no consistent correlation between mild to moderate eosinophilia on the father's side and the macular changes. No activity of fructose-1-phosphate aldolase was demonstrated in the sera of the proband, mother, father, and younger brother, Activity of this enzyme in other members of the family showed no relationship to the macular changes.

The authors thank Dr. P. Ellison of the Department of Ophthalmology for optic fundi photography and examination of several family members; Dr. B.Volk for performing fructose-1-phosphate aldolase determinations in his laboratories; Dr. S. Donahue for electron microscopy studies; Drs. R. Rohn and W. Bond for haematological studies; Dr. B. Hanna for advice on genetics, and Dr. A. L. Drew for encouragement and advice.

\section{REFERENCES}

Aronson, S. M., Lewitan, A., Rabiner, A. M., Epstein, N., and Volk, B. W. (1958). Arch. Neurol. Psychiat. (Chic.), 79, 151.

Aronson, B. E., and Volk, B. W. (1959). A.M.A. J. Dis. Child. 98, 50 .
- and Volk, B. W. (1962). In Cerebral Sphingolipidoses, edited by S. M. Aronson and B. W. Volk, p. 375. Academic Press, New York and London.

- - Perle, G., Saifer, A., and Volk, B. W. (1962). Proc. Soc. exp. Biol. (N.Y.), 3, 664.

Dunn, W. L., and Pearce, R. H. (1961). Canad. med. Ass. J., 84, 272.

Gomez, C. J., Gonatas, J., Korey, S. R., Samuels, S., Stein, A., Suzuki, K., Terry, R. D., and Weiss, M. (1963). J. Neuropath. exp. Neurol., 22, 1.

Knudson, A. G. and Kaplan, W. D. (1962). In Cerebral Sphingolipidoses, p. 395. Academic Press, New York and London.

Kozinn, P. J., Wiener, H., and Cohen, P. (1957). J. Pediat., 51, 58.

Lazarus, S. S., Wallace, B. J., and Volk, B. W. (1962). Amer. J. Path., $41,579$.

Maruyama, Y., Takeuchi, S., Tajiri, R., Harjano, S., and Makita, A. (1960). Med. J. Shinshu Univ., 5, 237.

Monnet, P., Tommasi, M., Tissot, A., and Gaillot. (1959). Arch. frans. Pediat., 16, 387.

Myrianthopoulos, N. C. (1962). In Cerebral Sphingolipidoses, p. 359. Academic Press, New York and London.

Spiegel-Adolf, M., Baird, H. W., Coleman, H. S., and Szekely, E. G. (1962). In Cerebral Sphingolipidoses, p. 129. Academic Press, New York and London.

Terry, R. D., and Korey, S. R. (1960). Nature (Lond.), 188, 1000. - Korey, S. R., and Weiss, M. (1962). In Cerebral Sphyngolipidoses, p. 49. Academic Press, New York and London.

Van Bogaert, L. (1962). World Neurol., 3, 512.

Walsh, F. B. (1957). Clinical Neuro-ophthalmology, 2nd ed., pp. 667-669, 674-675. Williams and Wilkins, Baltimore.

Wintrobe, M. M. (1961). Clinical Hematology, 5th ed., pp. 257-260, 581, 938-939. Lea \& Febiger, Philadelphia.

Wyburn-Mason, R. (1943). Brit. J. Ophthal., 27, 145 and 193. 